\title{
Accuracy of the Auto Scoring by the S9 CPAP in Patients with Obstructive Sleep Apnea
}

\author{
Joon Hyun Baek, MD, Ji-Ye Jeon, MD, Sang-Ahm Lee, MD \\ Department of Neurology, Asan Medical Center, University of Ulsan College of Medicine, Seoul, Korea
}

Background and Objective Several continuous positive airway pressure (CPAP) devices currently in use automatically estimate and provide information of the residual respiratory events such as apnea-hypopnea index (AHI), apnea index (AI), and hypopnea index (HI). To compare the auto scoring of the residual respiratory events using the S9 CPAP device with simultaneous manual scoring and identify factors that are associated with difference between auto scoring and manual scoring in patients with OSA.

Methods Patients with moderate to severe OSA titrated manually using S9 CPAP device were included. The correlation between auto scoring and manual scoring was assessed during an overnight standard in-hospital CPAP titration.

Results Eighty-six patients with moderate to severe OSA were included. There was a strong correlation between auto scoring and manual scoring on AHI $(\mathrm{r}=0.74, \mathrm{p}<0.001)$, with a stronger correlation on the $\mathrm{AI}(\mathrm{r}=0.86, \mathrm{p}<0.001)$, and a weaker correlation on $\mathrm{HI}(\mathrm{r}=0.56$, $\mathrm{p}<0.001$ ). Overall, S9 auto scoring tended to underestimate the AHI (mean AHI difference: -1.30 ) owing to the strong underestimation on HI. Higher BMI, higher AHI from diagnostic polysomnography, higher leakage and lower oxygen saturation were independent factors for greater difference between auto scoring and manual scoring.

Conclusions Auto scoring showed strong correlation with manual scoring. However, auto scoring of S9 CPAP tended to underestimate the AHI, as compared to manual scoring. Characteristic features of severe OSA were associated factors for difference between auto scoring and manual scoring.

Sleep Med Res 2016;7(1):26-32

Key Words Obstructive sleep apnea, Auto scoring, Manual scoring, S9 CPAP, AHI, Auto-CPAP.

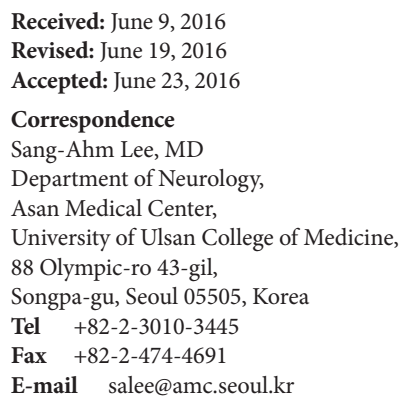

\section{INTRODUCTION}

Obstructive sleep apnea (OSA) is a highly prevalent, chronic, multisystem disease that can affect quality of life and increase health risk such as cardiovascular disease, hypertension, stroke and depression..$^{1-3}$ OSA could have serious consequences if left untreated. Continuous positive airway pressure (CPAP) treatment is established as a safe and effective therapy for OSA to suppress symptoms of apneas and hypopneas. ${ }^{4-7}$ It also reduces other complications and overall mortality in OSA patients. ${ }^{8}$

Currently, several CPAP devices are designed to automatically detect residual respiratory events and provide information about the residual apnea-hypopnea index (AHI). Estimated residual respiratory event data from CPAP device can be easily obtained by downloading data from the smartcard in place. Such auto scoring system allows evaluation of treatment efficiency in patients on in-home CPAP treatment.

Comparison between the residual AHI automatically scored by CPAP device and manually scored by the sleep technicians is previously reported. ${ }^{9,10}$ However, the technology and algorithm of respiratory event detection are different in each device and software. This results in significant differences in measures of residual respiratory events based on the CPAP machine used. For example, some previous studies showed that auto scoring by CPAP devices tend to overestimate the AHI, as compared to manual scoring of polysomnography (PSG) by sleep technicians. ${ }^{911,12}$ In contrast, Nigro et al. ${ }^{13}$ recently showed that $S 9$ auto-CPAP tends to underes- 
timate the AHI compared to manual scoring. Also, factors associated with differences between manual scoring and auto scoring have not been fully assessed. The aim of this study was to investigate the accuracy of the auto scoring during CPAP titration using S9 CPAP (S9 ResMed Corp., Sydney, Australia) and identify factors that affect differences between the auto scoring and manual scoring.

\section{METHODS}

\section{Subjects}

The study was cross-sectional in design. Patients included were adult patients who visited the sleep laboratory in order to undergo CPAP titration from July 2014 to April 2015. All patients were recruited from a single university hospital in Korea.

The inclusion criteria were as follows: 1) patients older than 18 years; 2) patients with moderate to severe sleep apnea, defined as an AHI of > 15; and 3) OSA patients newly diagnosed by the initial diagnostic PSG performed in the same laboratory. The exclusion criteria were as follows: 1) patients who had central sleep apnea $>50 \%$ of sleep apnea during the initial diagnostic PSG; 2) patients who underwent split-night studies or use of oxygen; 3 ) total sleep time $<180$ min on CPAP titration; and 4) patients who were intolerant to CPAP titration.

Age, sex, body mass index (BMI), neck circumference and Epworth Sleepiness Scale (ESS) were recorded at the time of the initial diagnostic PSG. Basic demographic information, medical comorbidities, and medication information were obtained from the self-reported checklist for medical history and electronic medical record. The Asan Medical Center Ethics Committee approved the study.

\section{Measurements}

OSA diagnoses were made using standard PSG (RemLogic version 2.0, Embla Systems Inc., Broomfield, CO, USA), which included electroencephalography, electrooculography, electromyography, electrocardiography, pulse oxymetry for oxygen saturation, and a microphone to detect snoring. Airflow was assessed using an oronasal thermistor and a nasal pressure transducer; and respiratory effort of thoracoabdominal movement was assessed using respiratory inductance plethysmography.

CPAP titration studies were performed by a sleep technologist during laboratory PSG using the S9 CPAP. CPAP was manually titrated to the lowest effective CPAP level based on clinical guidelines. ${ }^{14}$ The starting $\mathrm{CPAP}$ was $4 \mathrm{~cm} \mathrm{H}_{2} \mathrm{O}$, and the pressure was increased by at least $1 \mathrm{~cm} \mathrm{H}_{2} \mathrm{O}$ after an interval of $\geq 5 \mathrm{~min}$. CPAP was increased if there were 2 obstructive apneas, 3 hypopneas, or 5 respiratory-effort-related arousals, or at least 3 min of loud or unambiguous snoring. ${ }^{14}$ If the patient awoke and complained that the pressure was too high, it was restarted at a sufficiently comfortable lower pressure that allowed the patient to return to sleep. Down titration was not performed. The titrated CPAP level was defined as the lowest effective pressure.

Sleep and associated events were scored according to the American Academy of Sleep Medicine manual. ${ }^{15}$ The AHI was defined as the average number of episodes of apnea and hypopnea per hour. Apnea was defined as a drop in the peak thermal sensor excursion by at least $90 \%$ of baseline for at least $10 \mathrm{~s}$. A hypopnea was defined as a drop in the nasal pressure signal by at least $30 \%$ of baseline for at least $10 \mathrm{~s}$, with at least $4 \%$ reduction of $\mathrm{O}_{2}$ saturation from the pre-event baseline. The apnea in$\operatorname{dex}(\mathrm{AI})$ and the hypopnea index (HI) was calculated as the average number of apnea and hypopnea for each per hour. The AHI was used as an index of OSA severity. Percentage and time of each sleep period, total sleep time, oxygen saturation and leakage were also recorded. The CPAP device with the smart card in place automatically estimated residual AHI, AI, and $\mathrm{HI}$ using automatic analysis software version 3.16.

\section{Statistical Analysis}

The data were expressed as the mean \pm standard deviation (SD) for continuous variables or numbers and percentages for categorical variables. The automatically estimated $\mathrm{AHI}, \mathrm{AI}$ and $\mathrm{HI}$ were compared with the manually scored respiratory events during CPAP titration using Bland-Altman analysis, Wilcoxon signed rank test, intraclass correlation coefficient (ICC) and Spearman's coefficient of rank correlation due to non-Gaussian distribution. The Kruskal-Wallis test and Mann-Whitney test was used to compare between patients within and out of the limits of agreement. In order to examine independent factors associated with the difference between the manual scoring and the auto scoring, stepwise multiple regression analyses were performed on variables with $\mathrm{p}<0.05$ in univariate analysis. Age, sex, BMI, neck circumference, lowest oxygen saturation, sleep efficiency, total arousal index, AHI of the diagnostic PSG, degree of leakage (median, 95th percentile and maximum) and sleep percentages of $\mathrm{N} 1, \mathrm{~N} 2, \mathrm{~N} 3$, and rapid eye movement (REM) sleep were variables for greater difference between auto and manual scoring and within and out of limits of agreements. The level of statistical significance was set at $\mathrm{p}$ values $<0.05$. The statistical analysis was carried out using Statistical Package for Social Science Software (version 21.0; IBM SPSS Statistics for windows, Armonk, NY, USA; IBM Corporation).

\section{RESULTS}

Of the 92 patients initially evaluated, 4 were excluded due to unavailable results of initial diagnostic PSG and 2 were excluded due to intolerance of the CPAP titration. Thus, 86 patients provided acceptable data to evaluate the performance of S9 auto scoring. 


\section{Patient Characteristics}

A total of 86 patients (74 men, and 12 women) were enrolled. Among them, 59 (68.6\%) and 27 (31\%) patients had severe and moderate OSA, respectively. The average age was 51.2 years (SD 12.6 years). The mean AHI of diagnostic PSG was 43.6/h (SD 20.1/h) (Table 1). Table 2 showed PSG findings, oxygen saturation, and leakage data during CPAP titration night.

\section{Relationship and Agreement between Manual Scoring and Auto Scoring}

The mean manual-AHI was $5.3 \pm 3.8 / \mathrm{h}$; whereas, the mean auto-AHI was $4.0 \pm 3.8 / \mathrm{h}$. Auto-AHI was significantly lower than manual-AHI $(\mathrm{p}<0.001)$. However, there was a good correlation between manual-AHI and auto-AHI $(r=0.710$; $<<0.001)$ (Fig. 1A). A Bland and Altman plot showed that auto scoring tended to overestimate manual-AHI at low values of AHI and tended to underestimate manual AHI at higher AHI levels (Fig. 1B). The mean AHI difference was $-1.30 / \mathrm{h}$ and the limits of agreement for the AHI were from 3.99 to -6.60 .

The mean manual-AI was $1.6 \pm 2.2 / \mathrm{h}$; whereas, the mean au-

Table 1. Characteristics of participating patients

\begin{tabular}{lc}
\hline & Mean $\pm \mathrm{SD}$ \\
\hline Age, years & $51.2 \pm 12.6$ \\
Sex (male/female) & $76 / 12$ \\
Body mass index, $\mathrm{kg} / \mathrm{m}^{2}$ & $26.7 \pm 3.3$ \\
Epworth Sleepiness Scale & $9.6 \pm 5.1$ \\
Diagnostic PSG AHI (n/h) & $43.6 \pm 20.1$ \\
Hypertension & $37(43.0 \%)$ \\
Diabetes mellitus & $17(19.8 \%)$ \\
\hline
\end{tabular}

PSG: polysomnography, AHI: apnea-hypopnea index.

Table 2. Polysomnographic variables of patients during CPAP titration

\begin{tabular}{lc}
\hline & Mean \pm SD \\
\hline Sleep efficiency (\%) & $90.5 \pm 7.7$ \\
Total sleep time (min) & $376.2 \pm 36.6$ \\
Sleep stage, \% of TST & \\
N1 & $18.3 \pm 7.9$ \\
$\mathrm{~N} 2$ & $51.2 \pm 10.0$ \\
$\mathrm{~N} 3$ & $6.6 \pm 8.0$ \\
$\mathrm{REM}$ & $23.9 \pm 7.4$ \\
Lowest SO & \\
Leak, L/min & $88.0 \pm 4.6$ \\
Maximum & \\
95th percentile & $75.0 \pm 56.5$ \\
Median & $8.2 \pm 8.3$ \\
\hline
\end{tabular}

REM: rapid eye movement, TST: total sleep time, CPAP: continuous positive airway pressure.
to-AI was $3.2 \pm 3.4 / \mathrm{h}$. Auto-AI was significantly higher than manual-AI $(\mathrm{p}<0.001)$. However, there was also a strong correlation between the manual-AI and the auto-AI ( $\mathrm{r}=0.798$; $\mathrm{p}<$ 0.001) (Fig. 2A). A Bland and Altman plot showed that auto scoring tended to overestimate manual-AI at higher AI levels (Fig. $2 \mathrm{~B}$ ). The mean AI difference was 1.66/h and the limits of agreement for the AI were from 5.65 to -2.32 .

The mean manual-HI was $3.8 \pm 2.8 / \mathrm{h}$; whereas, the mean auto-HI was $0.8 \pm 0.6 / \mathrm{h}$. Auto-HI was significantly lower than manual-HI $(\mathrm{p}<0.001)$. There was modest correlation between the manual-HI and the auto-HI $(r=0.575 ; \mathrm{p}<0.001)$ (Fig. 3A). A Bland and Altman plot showed that auto scoring underestimated manual-HI at higher HI levels (Fig. 3B). The mean HI difference was $-2.98 / \mathrm{h}$ and the limits of agreement for the HI were from 1.98 to -7.94 . Especially, manual scoring of $\mathrm{HI}$ showed strong correlation with $\mathrm{HI}$ difference $(\mathrm{r}=-0.979 ; \mathrm{p}<0.001)$.

Table 3 summarized mean residual respiratory events of auto scoring and manual scoring each. Intraclass correlations between measures of residual respiratory events by auto scoring and manual scoring were calculated. The overall correlation coefficients were good when residual respiratory events were measured using $\mathrm{AHI}$, and $\mathrm{AI}$ compared to $\mathrm{HI}$.

\section{Comparison between Patients within and Out of the Limits of Agreement}

Four patients (4.6\%) exceeded the limits of agreement range for the AHI and 3 patients (3.5\%) were below the limits of agreement range for the AHI. Compared to the patients within the limits of range for the AHI, only patients group below the limits of agreement range for the AHI had higher BMI $(\mathrm{p}=0.02)$ and higher median leakage $(\mathrm{p}=0.008)$.

For the AI, 4 patients (4.6\%) exceeded the limits of agreement range. They had more severe OSA on the diagnostic PSG ( $\mathrm{p}=$ $0.010)$, lower lowest oxygen saturation $(p=0.003)$ and more $95 \%$ leakage ( $\mathrm{p}=0.025$ ) than patients within the limits of range for the AI.

Six patients (7.0\%) were below the limits of agreement range for the HI. They had more severe OSA on the diagnostic PSG ( $p=$ $0.002)$, lower lowest oxygen saturation $(p=0.020)$, more median leakage $(\mathrm{p}=0.012)$ and more REM portion $(\mathrm{p}=0.030)$ than patients within the limits of range for the HI.

\section{Factors Associated with Greater Differences between Auto Scoring and Manual Scoring}

Differences in residual respiratory events between automatic and manual scoring were significantly related to lower BMI and lower total arousal index on the diagnostic PSG with regard to AHI, lower lowest oxygen saturation and higher median leakage with regard to $\mathrm{AI}$, and lower total arousal index and higher lowest oxygen saturation with regard to HI (Table 4). 


\section{DISCUSSION}

In this study, we evaluated the accuracy of measures of residual respiratory events by auto scoring in OSA patients on CPAP titration and identified the factors that are associated with difference between auto scoring and manual scoring. Auto scoring of residual respiratory events of S9 CPAP were compared with simultaneous manual scoring of residual respiratory events during CPAP titration study. The main findings were as follows. 1) There were strong correlations between the residual respiratory events determined by the auto scoring of S9 CPAP device and manual scoring of PSG. 2) However, there were significant dif- ferences of the mean residual respiratory events. Auto scoring of S9 CPAP tended to underestimate the AHI and $\mathrm{HI}$ and overestimate the AI compared to manual scoring. 3) Accuracy of the auto scoring of the S9 CPAP was more reliable on less severe OSA. 4) Related factors of severe OSA were associated with greater difference between auto scoring and manual scoring.

Some studies have compared auto scoring of the residual AHI provided by several CPAP devices against the manual scoring of the residual AHI calculated from the PSG. ${ }^{9-13,16}$ Most of these studies showed strong correlation between the two methods even though the degree of agreement between these two methods differed based on the criterion used to define residual
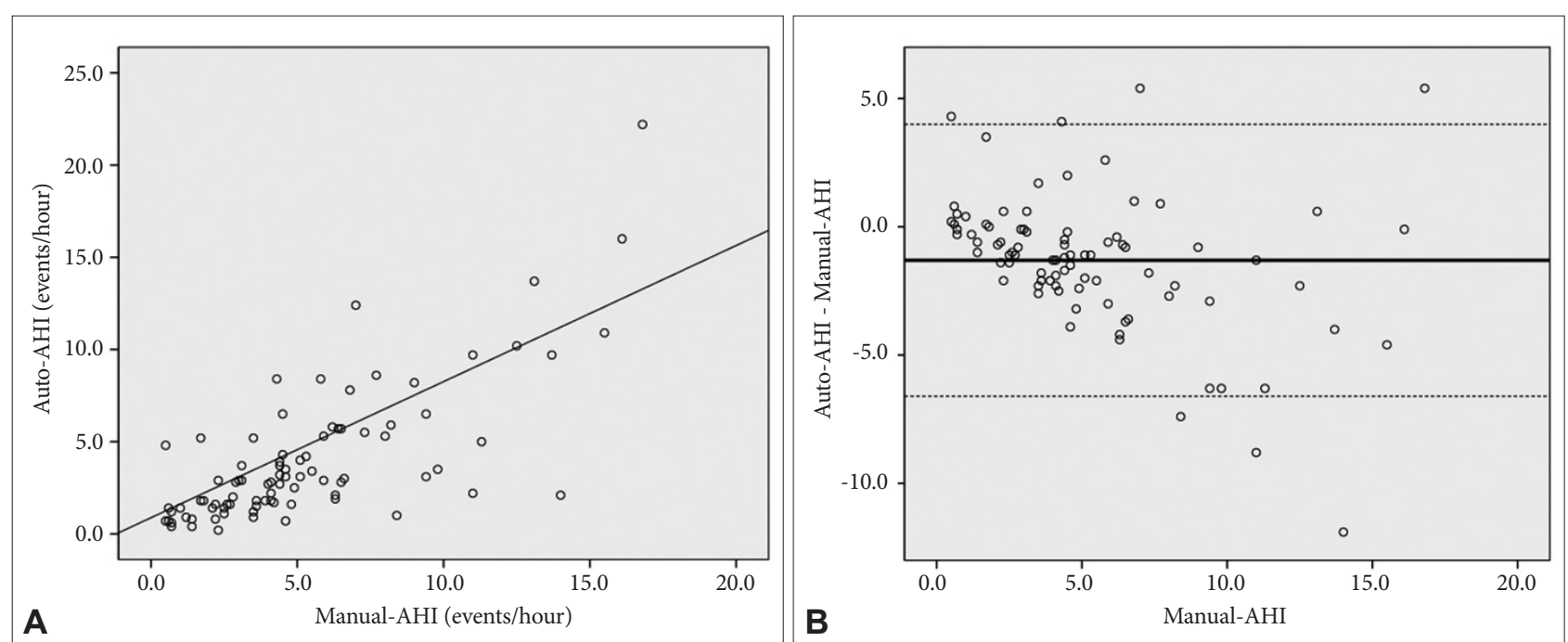

Fig. 1. A: Scatter plots of the apnea-hypopnea index (AHI) derived from the manual scoring (manual-AHI) and the AHI from the S9 CPAP device (auto-AHI). B: Bland-Altman plots according to the AHI. The Y-axis indicates the difference between the manual-AHI and the auto$\mathrm{AHI}[($ auto-AHI) - (manual-AHI)]. The X-axis indicates AHI determined by manual scoring. The solid line represents the mean difference; the dashed lines represent the limit of agreement ( $\pm 1.96 \mathrm{SD})$. CPAP: continuous positive airway pressure.
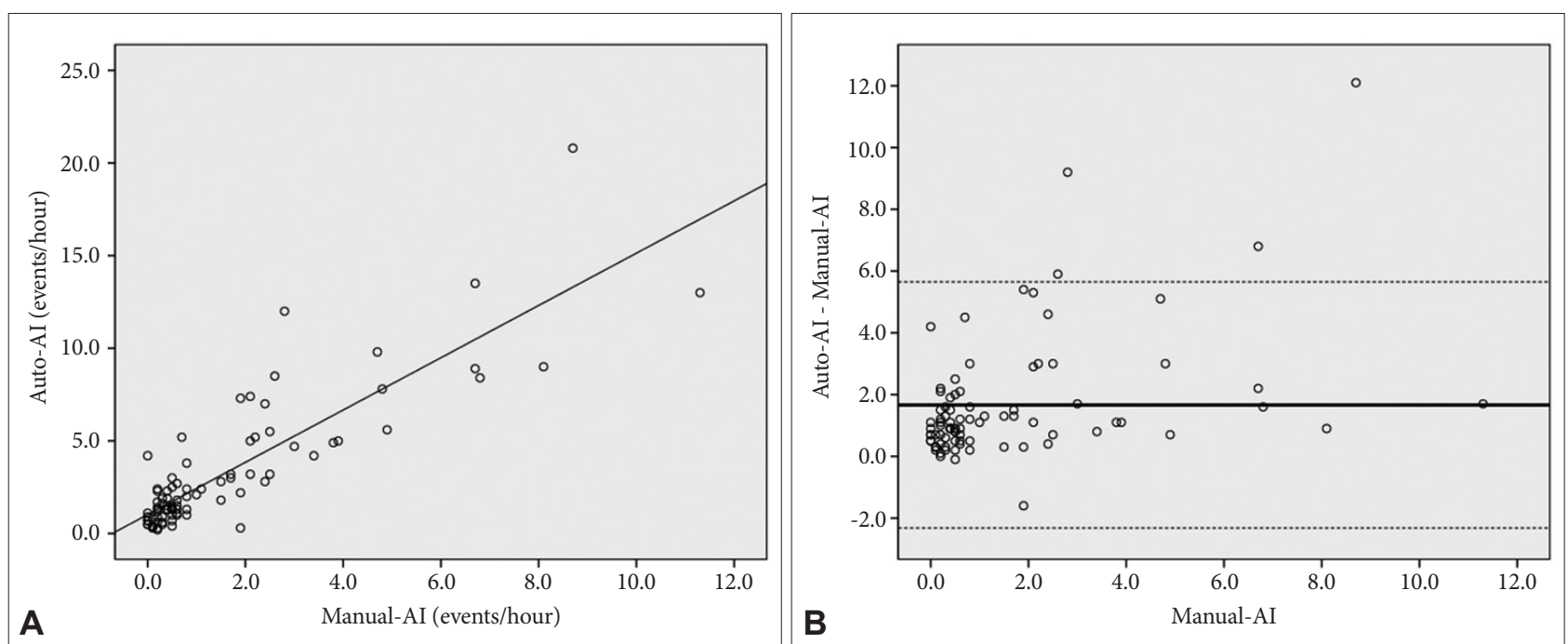

Fig. 2. A: Scatter plots of the apnea index (Al) derived from the manual scoring (manual-Al) and the Al from the S9 CPAP device (auto-AI). $\mathrm{B}$ : Bland-Altman plots according to the Al. The Y-axis indicates the difference between the manual-Al and the auto-Al [(auto-Al) - (manual$\mathrm{Al})$ ]. The $\mathrm{X}$-axis indicates Al determined by manual scoring. The solid line represents the mean difference; the dashed lines represent the limit of agreement ( $\pm 1.96 \mathrm{SD})$. CPAP: continuous positive airway pressure. 
respiratory events by the auto-CPAP devices. Results of our study indicated a strong correlation between the residual respiratory events determined by the auto scoring of S9 CPAP device and AHI derived from simultaneous manual scoring. Currently, auto-CPAP as well as fixed-CPAP has an inbuilt auto scoring system; hence, these results may have significant value in the clinical situation to assess treatment of respiratory events with the prescribed pressure of CPAP.

The results of our study differed with results of other similar studies. In our results, S9 auto scoring underestimated the AHI compared with manual scoring (the mean difference: -1.53 ). However, in most other studies, the auto scoring tended to
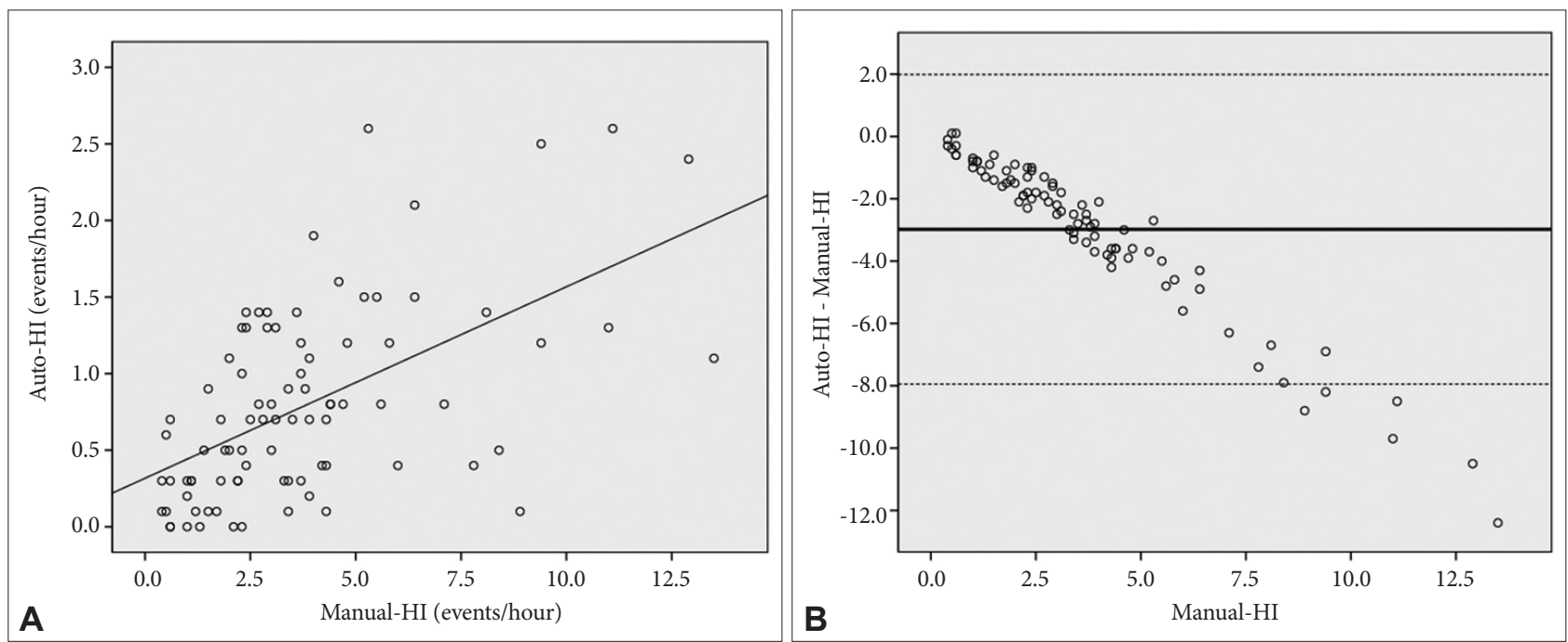

Fig. 3. A: Scatter plots of the hypopnea index $(\mathrm{HI})$ derived from the manual scoring (manual-HI) and the HI from the S9 CPAP device (auto$\mathrm{HI})$. B: Bland-Altman plots according to the HI. The Y-axis indicates the difference between the manual-HI and the auto-HI [(auto-HI) (manual-HI)]. The $\mathrm{X}$-axis indicates $\mathrm{HI}$ determined by manual scoring. The solid line represents the mean difference; the dashed lines represent the limit of agreement $( \pm 1.96 \mathrm{SD})$. CPAP: continuous positive airway pressure.

Table 3. Comparison of residual respiratory events between auto scoring and manual scoring

\begin{tabular}{lccr}
\hline & AHI & AI & HI \\
\hline Auto scoring $(\mathrm{n} / \mathrm{h})$ & $4.0 \pm 3.8$ & $3.2 \pm 3.6$ & $0.8 \pm 0.6$ \\
Manual scoring $(\mathrm{n} / \mathrm{h})$ & $5.3 \pm 3.8$ & $1.6 \pm 2.2$ & $3.8 \pm 2.8$ \\
Difference (n/h) & $-1.30 \pm 2.7$ & $1.66 \pm 2.0$ & $-2.98 \pm 2.5$ \\
ICC $(95 \% \mathrm{CI})$ & $0.744(0.632-0.825)$ & $0.765(0.661-0.840)$ & $0.238(0.029-0.428)$ \\
\hline
\end{tabular}

Auto scoring, manual scoring and difference are presented with mean \pm SD. Differences are calculated from (auto scoring measured by S9 auto CPAP) - (manual scoring measured by sleep technician).

AHI: apnea-hypopnea index, AI: apnea index, HI: hypopnea index, ICC: intraclass correlation coefficient, CI: confidence interval, CPAP: continuous positive airway pressure.

Table 4. Simple and multiple regression analysis for greater differences between auto scoring and manual scoring

\begin{tabular}{|c|c|c|c|c|c|}
\hline & & \multicolumn{2}{|c|}{ Simple } & \multicolumn{2}{|c|}{ Multiple } \\
\hline & & $\beta$ & $\mathrm{p}$ & $\beta$ & $\mathrm{p}$ \\
\hline \multirow[t]{2}{*}{ AHI } & BMI & -0.260 & 0.003 & -0.301 & $<0.001$ \\
\hline & Arousal index & -0.105 & 0.035 & -0.135 & 0.005 \\
\hline \multirow[t]{2}{*}{ AI } & Lowest $\mathrm{SO}_{2}$ & -0.234 & $<0.001$ & -0.207 & $<0.001$ \\
\hline & Leak median & 0.500 & 0.001 & 0.301 & 0.032 \\
\hline \multirow[t]{2}{*}{ HI } & Arousal index & -0.111 & 0.017 & -0.122 & 0.002 \\
\hline & Lowest $\mathrm{SO}_{2}$ & 0.248 & $<0.001$ & 0.152 & 0.006 \\
\hline
\end{tabular}

Differences between auto scoring and manual scoring are calculated from (auto scoring measured by $\$ 9$ auto CPAP) - (manual scoring measured by sleep technician).

BMI: body mass index, Arousal index: total arousal index, Lowest $\mathrm{SO}_{2}$ : lowest oxygen saturation, AHI: apnea-hypopnea index, AI: apnea index, HI: hypopnea index. 
overestimate the AHI compared to manual scoring at the expense of $\mathrm{HI} .{ }^{9,11,12}$ For example, Ueno et al. ${ }^{9}$ reported that $\mathrm{S} 8$ auto-CPAP, which is an older version of our device, overestimated the AHI compared with manual scoring of PSG (the mean difference: +5.7) due to frequent overestimation of the HI compared to the AI. They assumed that AHI overestimation could be related to the different approaches of detecting residual respiratory events between auto scoring of auto-CPAP and manual scoring or different analysis parameters used.

Several possible reasons could explain underestimation of AHI by auto scoring compared with manual scoring in our study. First, auto scoring tended to overestimate manual-AHI at low AHI values and underestimate manual AHI at higher AHI levels, as shown in most other studies. For example, Desai et al. ${ }^{12}$ reported that auto-CPAP device (Remstar Auto with software version Encore Pro 1.6 Respironics, Murrysville, PA, USA) estimate of average AHI overestimated PSG-AHI at low values of AHI and tended to underestimate PSG-AHI at higher AHI values. We enrolled more severe OSA patients (mean AHI from diagnostic PSG $=43.6 \pm 20.1$ ), as compared to other studies (mean AHI from diagnostic PSG $=26.3$ to 45.09 ) that additionally enrolled mild OSA patients..$^{11-13,16}$ Therefore, differences in patient selection could explain discrepancies between the study results. Second, technological differences in the S9 auto scoring of respiratory events may cause the underestimation of AHI. Interestingly, one recent prospective study with the same device showed that the S9 auto-CPAP had a tendency to underestimate the AHI, as compared to manual scoring of PSG. ${ }^{13}$ Thus, technological differences in the S9 auto-CPAP devices or different criteria used may have led to the underestimation of the respiratory events. Obstructive apnea was scored in the manual scoring as absence of airflow (thermistors) with the presence of respiratory effort; whereas, obstructive apnea was identified by the S9 auto scoring when a decrease in air flow was $>75 \%$ compared with recent average for $>10$ seconds associated with an increase in the upper airway resistance by forced oscillation technique. Hypopnea events were considered in the manual scoring when the respiratory signals (nasal pressure, thermistors) fell at least 30\% accompanied by oxygen desaturation or arousal, as compared to the $\mathrm{S} 9$ auto scoring of hypopnea on $50 \%$ nasal pressure drop compared with the recent average for $>10 \mathrm{sec}-$ onds. Therefore, $30 \%$ reduction of the nasal pressure can be checked more often than the 50\% reduction of nasal pressure; and $75 \%$ of the reduction of the airflow can be more easily checked compared to the absence of the airflow. Thus, S9 auto scoring missed many hypopneas and also misidentified many hypopneas as apneas. Accordingly, underestimation of $\mathrm{HI}$ can be considered as false negative and overestimation of AI can be considered as false positive in the auto scoring. Overall, the underestimation of hypopnea is larger than overestimation of apnea, and the AHI that is the sum of $\mathrm{HI}$ and $\mathrm{AI}$ would be lower compared to manual scoring.
In our study, there were tendency of greater differences between auto scoring and manual scoring at higher AHI levels. Higher BMI, higher AHI from the diagnostic PSG, higher 95\% leakage, higher median leakage and lower lowest oxygen saturation were associated factors with out of the limits of agreement compared to within the limits of agreement on AHI, AI and HI, all of which are characteristic features of severe OSA and higher CPAP pressure. ${ }^{17-20}$ Likewise, factors associated with greater difference between auto scoring and manual scoring were higher BMI, higher total arousal index, higher median leakage and lower lowest oxygen saturation. ${ }^{17-22}$ Considering that variables associated with greater overestimation of $\mathrm{AI}$ and underestimation of AHI and $\mathrm{HI}$ by auto scoring are characteristics of severe OSA and high CPAP pressure, more severe OSA could result in greater difference between auto scoring and manual scoring. Therefore, great caution is needed when assessing treatment effectiveness of CPAP using auto scoring, especially in severe OSA patients. Similarly, in the other study, greater AHI-PSG and greater 95th percentile leakage were associated with discrepancy of AHI between auto scoring and manual scoring. ${ }^{9}$ Further investigation is needed to evaluate independent factors associated with a discrepancy in the AHI scored by the 2 methods.

There were some limitations in our study. First, this study was conducted in an attended clinical setting; therefore, the overestimation of respiratory events by auto scoring due to the mask leakage rarely occurs. However, cautious interpretation is required since unattended auto scoring of CPAP could result in overestimation of respiratory events due to the leakage problem. Second, our results should not be applied to other autoCPAP devices. These different devices may have different algorithms and sensitivities to detect respiratory events. This study was performed with only one specific model of CPAP. For example, Kessler et al. ${ }^{23}$ showed that two types of auto-CPAP devices (AutoSet and Somnosmart) predicted significantly different therapeutic pressures for fixed CPAP therapy. Also, Stammnitz et al. ${ }^{24}$ showed considerable differences in the efficacy of the three devices (AutoSet, Horizon, Virtuoso). Third, this study did not subdivide the respiratory events into obstructive and central type, despite possible differences in correlation among the types of respiratory events. For example, Nigro et al. ${ }^{13}$ showed a moderate to good agreement (ICC $0.6-0.8$ ) between the total number of apneas or central apneas identified by the auto-CPAP and PSG. However, there were a very low agreement (ICC < $0.3)$ between auto-CPAP vs. PSG to recognize the obstructive apneas or hypopneas. Also, there is a possibility that residual respiratory events are central and not of obstructive type, with emergence of complex sleep apnea. In this situation, residual respiratory events by auto scoring could not be regarded as the result of insufficient CPAP treatment and thus, caution is needed in assessing the treatment effectiveness. Fourth, this is the study of highly select patients without significant comorbidities, hypoventilation syndromes, and central sleep apnea. There- 
fore, the study population is considered as a biased population among patients with sleep-disordered breathing. Lastly, most of the patients in our study had moderate to severe OSA with relatively lower BMI, so the results should be extrapolated to all OSA patients with caution.

In conclusion, a strong correlation and acceptable diagnostic accuracy between the auto scoring of the S9 CPAP and the manual scoring was observed. However, S9 auto scoring tended to underestimate the residual respiratory events compared to the manual scoring due to technical and criteria differences. In addition, auto scoring and manual scoring show greater differences in severe OSA patient compared to moderate OSA patient and the characteristics of severe OSA may additionally affect these differences. These results may guide clinicians who are planning to use the auto scoring of the CPAP for assessing treatment effectiveness.

\section{Acknowledgments}

This report was supported by the Asan Medical Center in 2016.

\section{Conflicts of Interest}

The authors have no financial conflicts of interest.

\section{REFERENCES}

1. Peppard PE, Young T, Palta M, Skatrud J. Prospective study of the association between sleep-disordered breathing and hypertension. $N$ Engl J Med 2000;342:1378-84.

2. Lavie P, Herer P, Hoffstein V. Obstructive sleep apnoea syndrome as a risk factor for hypertension: population study. BMJ 2000;320:479-82.

3. Schröder CM, O’Hara R. Depression and obstructive sleep apnea (OSA). Ann Gen Psychiatry 2005;4:13.

4. Sullivan CE, Issa FG, Berthon-Jones M, Eves L. Reversal of obstructive sleep apnoea by continuous positive airway pressure applied through the nares. Lancet 1981;1:862-5.

5. Engleman HM, Martin SE, Deary IJ, Douglas NJ. Effect of continuous positive airway pressure treatment on daytime function in sleep apnoea/hypopnoea syndrome. Lancet 1994;343:572-5.

6. Meurice JC, Paquereau J, Neau JP, Caron F, Dore P, Ingrand P, et al. Long-term evolution of daytime somnolence in patients with sleep apnea/hypopnea syndrome treated by continuous positive airway pressure. Sleep 1997;20:1162-6.

7. Kawahara S, Akashiba T, Akahoshi T, Horie T. Nasal CPAP improves the quality of life and lessens the depressive symptoms in patients with obstructive sleep apnea syndrome. Intern Med 2005;44:422-7.

8. Marin JM, Carrizo SJ, Vicente E, Agusti AG. Long-term cardiovascular outcomes in men with obstructive sleep apnoea-hypopnoea with or without treatment with continuous positive airway pressure: an observational study. Lancet 2005;365:1046-53.

9. Ueno K, Kasai T, Brewer G, Takaya H, Maeno K, Kasagi S, et al. Evaluation of the apnea-hypopnea index determined by the S8 auto-CPAP, a continuous positive airway pressure device, in patients with obstructive sleep apnea-hypopnea syndrome. J Clin Sleep Med 2010;6:146-51.

10. Prasad B, Carley DW, Herdegen JJ. Continuous positive airway pressure device-based automated detection of obstructive sleep apnea compared to standard laboratory polysomnography. Sleep Breath 2010;14: 101-7.

11. Ikeda Y, Kasai T, Kawana F, Kasagi S, Takaya H, Ishiwata S, et al. Comparison between the apnea-hypopnea indices determined by the REMstar Auto M series and those determined by standard in-laboratory polysomnography in patients with obstructive sleep apnea. Intern Med 2012;51:2877-85.

12. Desai H, Patel A, Patel P, Grant BJ, Mador MJ. Accuracy of autotitrating CPAP to estimate the residual Apnea-Hypopnea Index in patients with obstructive sleep apnea on treatment with autotitrating CPAP. Sleep Breath 2009;13:383-90.

13. Nigro CA, González S, Arce A, Aragone MR, Nigro L. Accuracy of a novel auto-CPAP device to evaluate the residual apnea-hypopnea index in patients with obstructive sleep apnea. Sleep Breath 2015;19:569-78.

14. Gugger M, Mathis J, Bassetti C. Accuracy of an intelligent CPAP machine with in-built diagnostic abilities in detecting apnoeas: a comparison with polysomnography. Thorax 1995;50:1199-201.

15. Berry RB, Budhiraja R, Gottlieb DJ, Gozal D, Iber C, Kapur VK, et al. Rules for scoring respiratory events in sleep: update of the 2007 AASM Manual for the Scoring of Sleep and Associated Events. Deliberations of the Sleep Apnea Definitions Task Force of the American Academy of Sleep Medicine. J Clin Sleep Med 2012;8:597-619.

16. Cilli A, Uzun R, Bilge U. The accuracy of autotitrating CPAP-determined residual apnea-hypopnea index. Sleep Breath 2013;17:189-93.

17. Hongyo K, Ito N, Yamamoto K, Yasunobe Y, Takeda M, Oguro R, et al. Factors associated with the severity of obstructive sleep apnea in older adults. Geriatr Gerontol Int 2016 Jun 1 [Epub ahead of print]. http:// dx.doi.org/10.1111/ggi.12768.

18. Sopkova Z, Dorkova Z, Tkacova R. Predictors of compliance with continuous positive airway pressure treatment in patients with obstructive sleep apnea and metabolic syndrome. Wien Klin Wochenschr 2009;121: 398-404.

19. Woehrle H, Graml A, Weinreich G. Age- and gender-dependent adherence with continuous positive airway pressure therapy. Sleep Med 2011;12:1034-6.

20. Montesi SB, Bakker JP, Macdonald M, Hueser L, Pittman S, White DP, et al. Air leak during CPAP titration as a risk factor for central apnea. $J$ Clin Sleep Med 2013;9:1187-91.

21. Pang KP, Terris DJ, Podolsky R. Severity of obstructive sleep apnea: correlation with clinical examination and patient perception. Otolaryngol Head Neck Surg 2006;135:555-60.

22. Lam JC, Xu A, Tam S, Khong PI, Yao TJ, Lam DC, et al. Hypoadiponectinemia is related to sympathetic activation and severity of obstructive sleep apnea. Sleep 2008;31:1721-7.

23. Kessler R, Weitzenblum E, Chaouat A, Iamandi C, Alliotte T. Evaluation of unattended automated titration to determine therapeutic continuous positive airway pressure in patients with obstructive sleep apnea. Chest 2003;123:704-10.

24. Stammnitz A, Jerrentrup A, Penzel T, Peter JH, Vogelmeier C, Becker HF. Automatic CPAP titration with different self-setting devices in patients with obstructive sleep apnoea. Eur Respir J 2004;24:273-8. 